\title{
Charged Particles Multiplicity and Scaling Violation of Fragmentation Functions in Electron-Positron Annihilation
}

\author{
Tooraj Ghaffary \\ Department of Science, Shiraz Branch, Islamic Azad University, Shiraz, Iran \\ Correspondence should be addressed to Tooraj Ghaffary; tooraj.gh@gmail.com
}

Received 30 April 2016; Revised 7 July 2016; Accepted 25 July 2016

Academic Editor: Andrea Coccaro

Copyright (c) 2016 Tooraj Ghaffary. This is an open access article distributed under the Creative Commons Attribution License, which permits unrestricted use, distribution, and reproduction in any medium, provided the original work is properly cited. The publication of this article was funded by $\mathrm{SCOAP}^{3}$.

\begin{abstract}
By the use of data from the annihilation process of electron-positron in AMY detector at $60 \mathrm{GeV}$ center of mass energy, charged particles multiplicity distribution is obtained and fitted with the KNO scaling. Then, momentum spectra of charged particles and momentum distribution with respect to the jet axis are obtained, and the results are compared to the different models of QCD; also, the distribution of fragmentation functions and scaling violations are studied. It is being expected that the scaling violations of the fragmentation functions of gluon jets are stronger than the quark ones. One of the reasons for such case is that splitting function of quarks is larger than splitting function of gluon.
\end{abstract}

\section{Introduction}

Hadron production in high energy interactions can be described by the parton cascade [1] (the propagation of gluons and their separation into partons), and it is not possible to describe the formation of hadrons through a perturbative description. Gluon radiation, as a prominent process in the parton cascade, is proportional to the color coefficient of radiated gluon coupling. This coefficient equals $C_{A}=3$ when it radiates gluon, but when it radiates quarks, it will be $C_{F}=4 / 3$ [2]. As a result, the multiplicity of soft gluons from a gluons source is about 9/3 times bigger than the multiplicity of quark source. Inequality of $C_{A}$ and $C_{F}$ plays an important role in the explanation of the observed differences between the gluon and quark jets. Compared with quarks' jets, it is being observed that gluon jets have higher width, more multiplicity, softer fragmentation functions, and stronger scaling violations of fragmentation functions [3]. The fragmentation function " $D_{a}^{h}\left(x, Q^{2}\right)$ " shows the possibility that a parton " $a$," which is being produced in the short distances, might be in the range of $1 / Q$ and fragment into a hadron " $h$ " which has a fraction " $x$ " of the momentum of the parton " $a$ " [4-7]. In LEP experiments, momentum fraction is
$x_{E}=E_{h} / E_{\text {jet }}$ in which $E_{h}$ shows the hadron energy of " $h$ " and $E_{\text {jet }}$ refers to a jet energy which $E_{h}$ belongs to [3].

Relative softness of fragmentation function of gluon jets in the range of small $x_{E}$ is being expressed by the multiplicity of radiated soft gluons; but, for higher values of $x_{E}$, it is being described by the fact that gluons cannot exist as valence partons inside a produced parton. The strong scaling violation of fragmentation functions of gluon's jet resulted from this fact as the dependency of this scale (means the fragmentation functions of gluon jet by the separation function of $P_{g \rightarrow g g} \sim C_{A}$ ) is prominent; however, the dependency of fragmentation functions of quark jet by the separation function of $P_{q \rightarrow q g} \sim C_{F}$ is dominant. Given that the momentum distribution of charged particles has a key role in the scaling violation of fragmentation functions, so, in this article, first charged particles distribution will be obtained and will be fitted with the KNO scaling [8] in order to determine if it is consistent with other data from other energies. Then, momentum distribution with respect to the jet axis will be obtained and, at the end, the distribution of the fragmentation functions will be considered. Moreover, by using the data collected by the AMY detector at $60 \mathrm{GeV}$ center of mass energy and comparing them with the ones obtained 


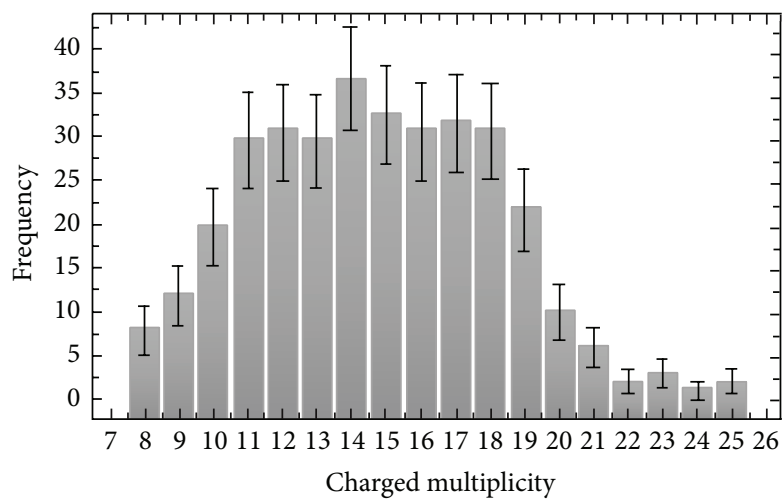

FIGURE 1: Frequency of charged particles multiplicity due to AMY data.

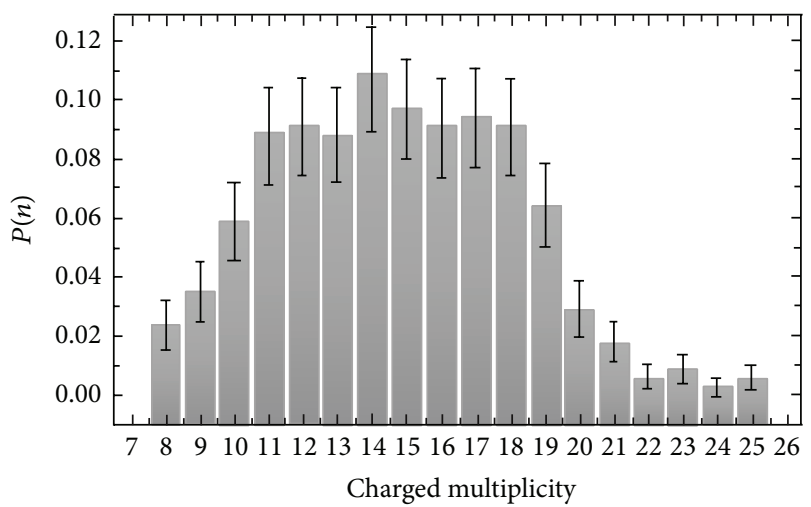

FIGURE 2: Probability distribution of charged particles multiplicity due to AMY data.

in other energy intervals, scaling violations of fragmentation functions will be discussed.

\section{Experimental Setup}

The AMY detector at Tristan was a multipurpose detector optimized for lepton identification. It was made up of several components, among which tracking chambers and a shower counter inside a 3-T solenoid magnet that allowed the detector to be compact while maintaining good momentum resolution. Charged particles are detected efficiently over the polar angle region $\cos \theta<0.87$ with a momentum resolution $\Delta p_{T}=0.7 \% \times\left\lfloor p_{T}(\mathrm{GeV} / c)\right\rfloor$. The detailed description of the various detector components has been described in [16].

\section{Multiplicity of Charged Particles}

Multiplicity distribution of charged particles measured by the AMY experiment is shown in Figure 1. Figure 2 shows the probability distribution of charged particles which indicated the cross section.

The average value of multiplicity of charged particles in AMY data is $14.68 \pm 3.83$, consistent with other data in other energy intervals. The average value increases with increasing of the energy [8, 17-19].

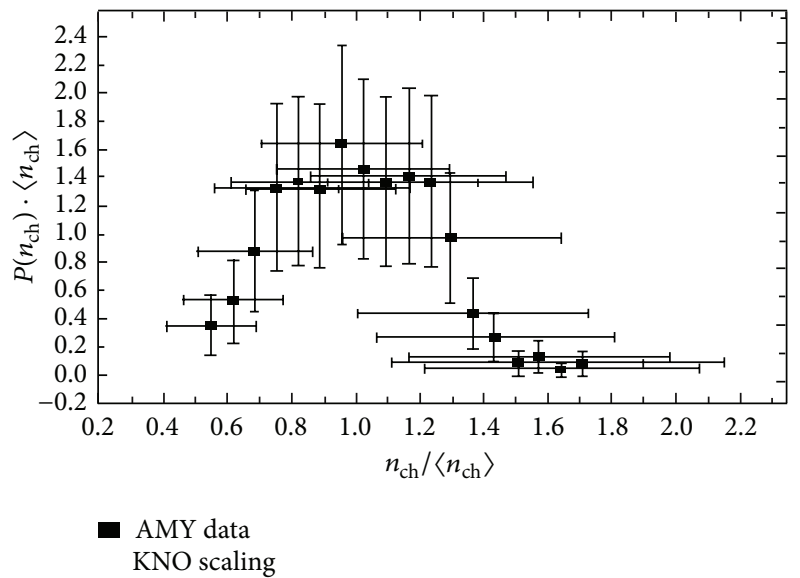

FIgURE 3: Measured charged particles multiplicity based on KNO scaling.

\section{KNO Scaling}

An interesting description for the distribution of multiplicities at a given energy was introduced by Koba, Nielsen, and Olesen (KNO) which was derived from Feynman scaling [20, 21]. The product of the mean charged particles multiplicity, $\left\langle n_{\mathrm{ch}}\right\rangle$, and the probability $P\left(n_{\mathrm{ch}}\right)$ for " $n_{\mathrm{ch}}$ " charged particles in the final state is given by a universal function defined as follows:

$$
\Psi\left(\frac{n_{\mathrm{ch}}}{\left\langle n_{\mathrm{ch}}\right\rangle}\right)=P\left(n_{\mathrm{ch}}\right)\left\langle n_{\mathrm{ch}}\right\rangle .
$$

For AMY data, this distribution can be drawn as a function of $n_{\mathrm{ch}}$ and it is shown in Figure 3.

Figure 3 suggests that there is an acceptable consistency with data taken in other energy ranges $[22,23]$. The scale function of KNO is being defined as follows [24]:

$$
\Psi(z)=\frac{k^{k}}{\Gamma(k)} z^{k-1} e^{-k z},
$$

where $z=n /\langle n\rangle$ and $\Gamma(k)$ is Gamma function. A fit of the AMY data to KNO function was performed and its result is shown in Figure 4.

For $\chi^{2} / N_{\mathrm{DF}}=0.799$, “ $k$ " equals $14.62 \pm 2.98$. Based on the existing errors in the tests, it can be shown that the value of " $k$ " for AMY data is consistent with the value of the other tests. The magnitude of " $k$ " has an interval between 11.64 and $17.60[16]$.

\section{Momentum Spectra of Charged Particles}

One of the features which is very effective in the interpretation of scaling violation is the effect of transverse momentum or $P_{T}$ on the fragmentation functions. For this reason, first, momentum spectra of charged particles to the jet axis for AMY data will be studied. We separate two and three jet events using the DURHAM [25] and JADE [26] jet clustering algorithm. In these algorithms the scaled mass 


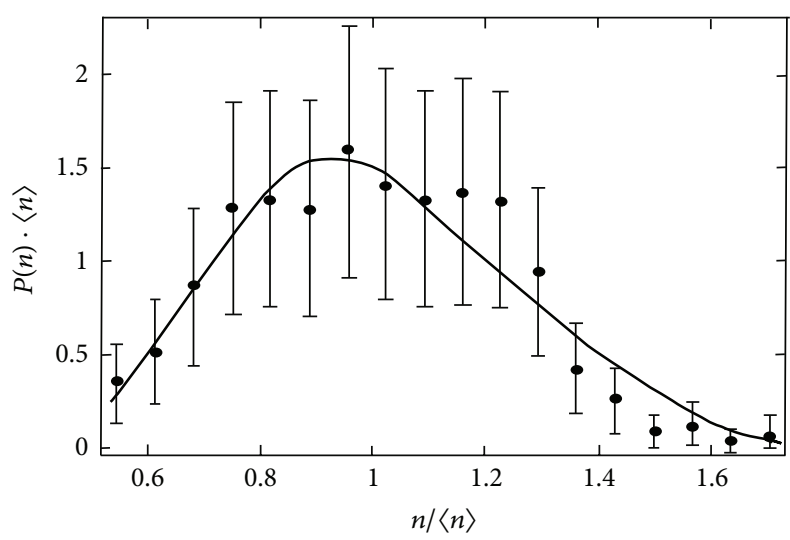

- AMY data

- KNO fit

FIGURE 4: Charged multiplicity distribution due to AMY data based on KNO scaling.

spread, defined as $Y_{i j}=m_{i j}^{2} / E_{\mathrm{vis}}^{2}$ with $m_{i j}=2 E_{i} E_{j}\left(1-\cos \theta_{i j}\right)$, is calculated for each pair of particles in the event where $E_{i}$, $E_{j}$ denote the energies, $\theta_{i j}$ is the angle between two objects $i, j$ under consideration, and $E_{\mathrm{vis}}^{2}$ is the squared invariant mass of the hadronic final state. If the smallest of $Y_{i j}$ values is less than a parameter $Y_{\text {cut }}$, the corresponding pair of particles is combined into a cluster by summing the four momenta. This process is repeated, using all combinations of clusters and remaining particles, until all $Y_{i j}$ values exceed $Y_{\text {cut }}$. The clusters remaining at this stage are defined as the jets. In this paper, JADE algorithm is used for finding jets. Using sphericity, the eigenvalues and eigenvectors of momentum tensor have been calculated [27]. A plane which is formed by the two eigenvectors corresponding to the two bigger eigenvalues of momentum tensor, $\left(\widehat{n}_{1}-\widehat{n}_{2}\right)$, is called event plane. The average of the transverse momentum in this plane, $\left\langle P_{T_{\text {in }}}\right\rangle$, is presented as follows:

$$
\left\langle P_{T_{\text {in }}}\right\rangle=\frac{1}{N_{\mathrm{ch}}} \sum_{i} \vec{P}_{i} \cdot \widehat{n}_{2}
$$

The summation is over on the charged particles. The average of the transverse momentum in the direction perpendicular to the event plane, $\left\langle P_{T_{\text {out }}}\right\rangle$, was presented as the following equation:

$$
\left\langle P_{T_{\text {out }}}\right\rangle=\frac{1}{N_{\text {ch }}} \sum_{i} \vec{P}_{i} \cdot \widehat{n}_{1}
$$

The summation is over on the charged particles. So, the average of total transverse momentum will be as follows:

$$
\left\langle P_{T}\right\rangle=\sqrt{\left\langle P_{T_{\text {in }}}\right\rangle^{2}+\left\langle P_{T_{\text {out }}}\right\rangle^{2}} .
$$

In Figure $5,\left(1 / \sigma_{\text {tot }}\right)(d \sigma / d p)$ related to the charged particles from AMY data in the energy of $60 \mathrm{GeV}$ along with results from the lower energies in the various experiments are shown.

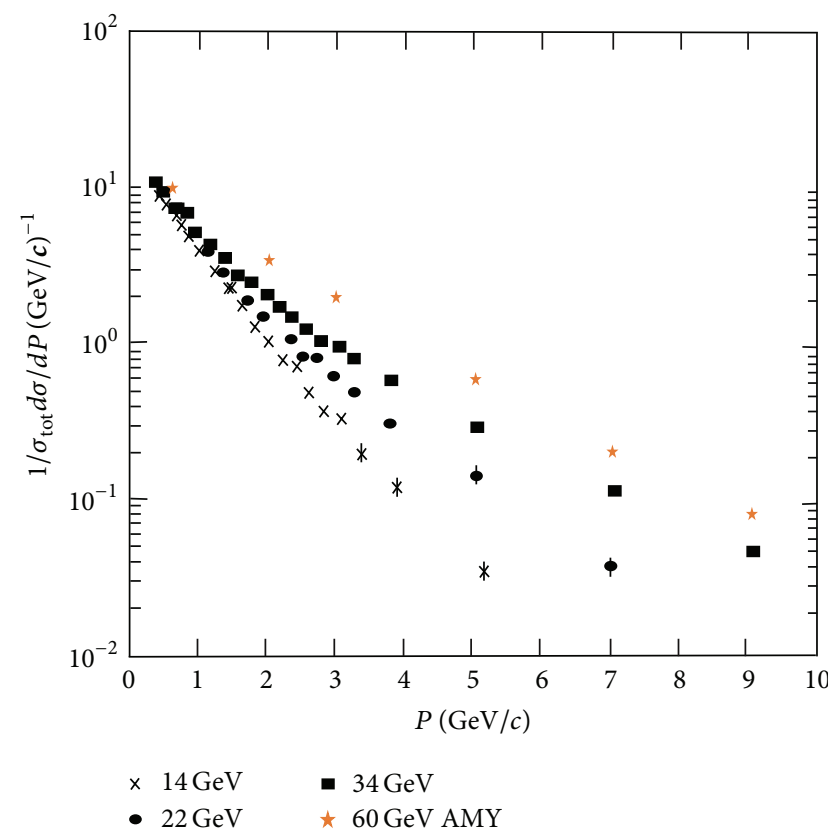

FIGURE 5: Normalized differential cross section as a function of the momentum of the charged particles for different center of mass energies [9].

Based on Figure 5, it can be seen that the cross section of particles' production for AMY data as well as the other data for $p>0.2$ will be decreased by increasing the momentum. So, by increasing energy, the widest curve will be shown by the momentum distribution. By decreasing the angle between two jets, the similarity of the event of three jets will be increased to the other event with two jets. One simple way for observing the gradual transfer (from the event of three jets into the event of two jets) is to look at the average distribution of transverse momentum. The average of the squared transverse momentum, in the event plane and perpendicular to this plane, is presented as follows:

$$
\begin{aligned}
& \left\langle P_{T_{\text {in }}}^{2}\right\rangle=\frac{1}{N_{\mathrm{ch}}} \sum_{i}\left(\vec{P}_{i} \cdot \widehat{n}_{2}\right)^{2}=Q_{2}\left\langle P^{2}\right\rangle \\
& \left\langle P_{T_{\text {out }}}^{2}\right\rangle=\frac{1}{N_{\mathrm{ch}}} \sum_{i}\left(\vec{P}_{i} \cdot \widehat{n}_{1}\right)^{2}=Q_{1}\left\langle P^{2}\right\rangle .
\end{aligned}
$$

In Figure 6, the average of the squared transverse momentum in the event plane, $\left\langle P_{T_{\text {in }}}^{2}\right\rangle$, and the average of the squared transverse momentum perpendicular to the event plane, $\left\langle P_{T_{\text {out }}}^{2}\right\rangle$, for AMY data along with the final results from different other energies are shown.

It is clear that $\left\langle P_{T_{\text {in }}}^{2}\right\rangle$ will increase much more strongly with respect to $\left\langle P_{T_{\text {out }}}^{2}\right\rangle$. The results of QCD model regarding gluon radiation and without such radiation are shown in Figure 6. It is being observed that AMY results with gluon radiation in QCD model are associated with more consistency. Finally, it can be concluded that data show the possibility of having gluon radiation at high energies. Thus studying the transverse momentum distribution of charged particles 


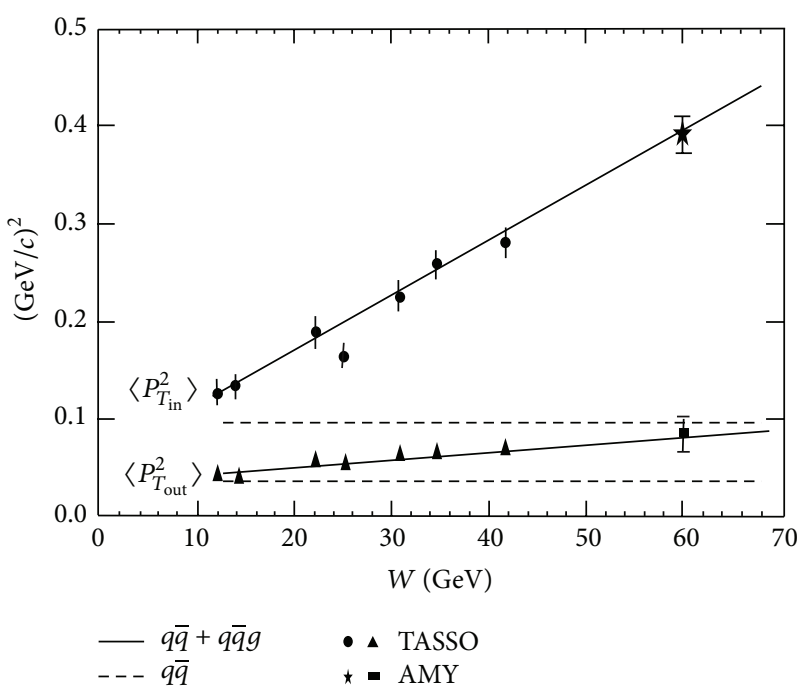

FIgURE 6: The average of the squared transverse momentum in the event plane, $\left\langle P_{T_{\text {in }}}^{2}\right\rangle$, and perpendicular to the event plane, $\left\langle P_{T_{\text {out }}}^{2}\right\rangle$, at different c.m. energies [10].

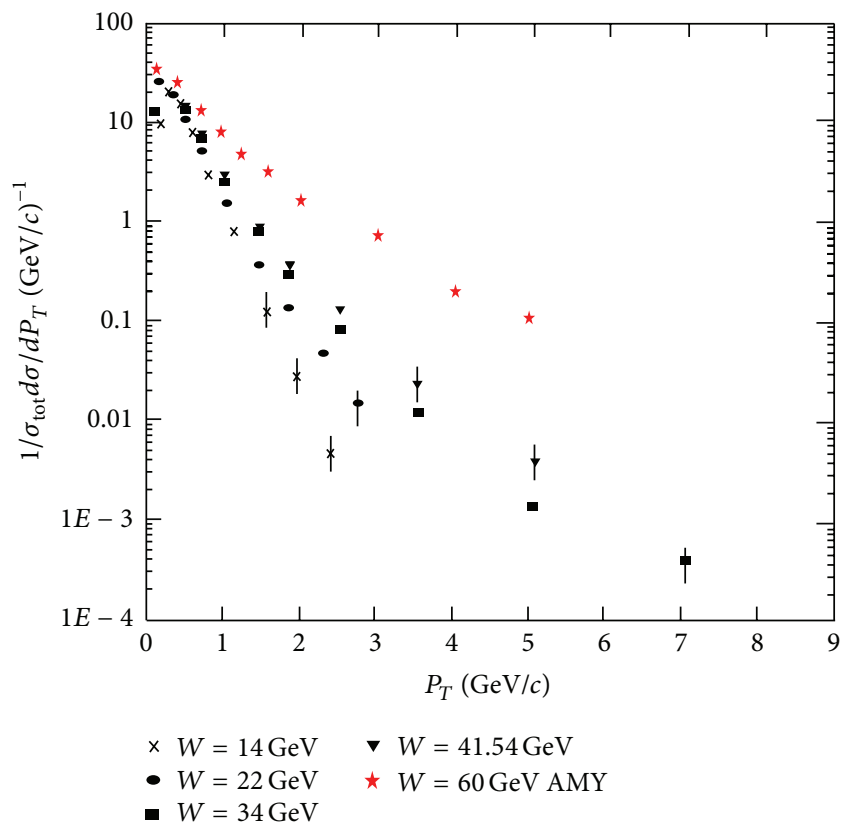

FIGURE 7: Normalized differential cross section as a function of the transverse momentum of the charged particles, $\left(\left(1 / \sigma_{\text {tot }}\right)\left(d \sigma / d P_{T}\right)\right)$, for different center of mass energies [11].

to the jet axis is quite important. Here, sphericity axis was selected as the jet one. In Figures 7 and 8 the two normalized differential cross sections as a function of the transverse momentum of the charged particles, $\left(\left(1 / \sigma_{\text {tot }}\right)\left(d \sigma / d P_{T}\right)\right)$, and of the squared transverse momentum of the charged particles, $\left(\left(1 / \sigma_{\text {tot }}\right)\left(d \sigma / d P_{T}^{2}\right)\right)$, are shown for different center of mass energies. For AMY data, $P_{T}$ and $P_{T}^{2}$ distributions in comparison to the data resulting from the other experiments will be studied. It is being expected that, by increasing energy, multiplicity of particles will increase [28]. So, increasing the

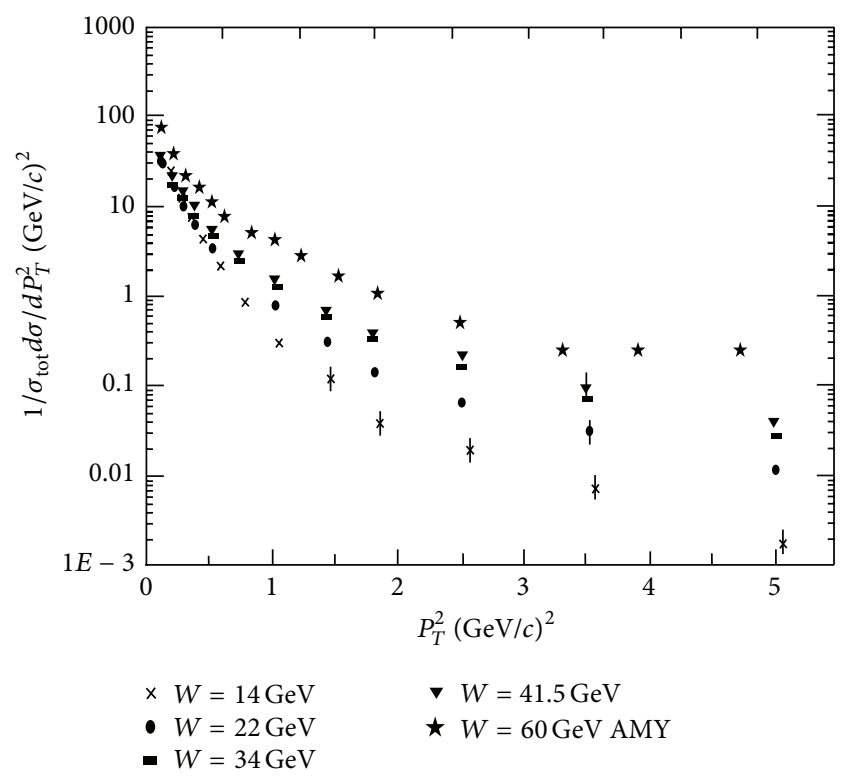

FIgURE 8: Normalized differential cross section as a function of the squared transverse momentum of the charged particles, $\left(\left(1 / \sigma_{\text {tot }}\right)\left(d \sigma / d P_{T}^{2}\right)\right)$, for different center of mass energies [10].

number of particles in $P_{T} \geq 0.5$, by increasing $W$, can be the reason of hard gluon radiation. In other words, this kind of radiation affects the flux of particles.

Now, the distribution of fragmentation functions and scaling violations will be studied.

\section{Distribution of Fragmentation Functions and Scaling Violation}

The fragmentation function is being defined as the whole number of charged particles, $N_{\mathrm{ch}}$, in the bin related to $x_{E}$ and $Q$ scale normalized to the number of jets “ $N_{\text {jet }}(Q)$ " [12, 29]:

$$
\frac{1}{N_{\text {jet }}(Q)} \frac{d N_{\mathrm{ch}}\left(x_{E}, Q\right)}{d x_{E}} \text {. }
$$

In this equation, $x_{E}$ shows

$$
x_{E}=\frac{E_{h}}{E_{\text {jet }}} .
$$

A simple pattern of momentum distribution in terms of energy is important; then the energy of particles is being scaled as $x=2 E_{h} / W$. In such case, the possibility of using this variable was suggested by Feynman. In the parton model, the cross section of $e^{+} e^{-} \rightarrow h+X$ is being defined by the following equation:

$$
\frac{1}{\sigma_{\text {tot }}} \frac{d \sigma}{d x}=\frac{1}{\sum e_{q}^{2}} \sum e_{q}^{2}\left[D_{q}^{h}(x, s)+D_{\bar{q}}^{h}(x, s)\right] .
$$

In this domain, the summation is over on all quarks which are being produced at the center of mass energy and $e_{q}$ is the quark charge. $D_{q}^{h}(x, s)$ defines a method in which the quarks 


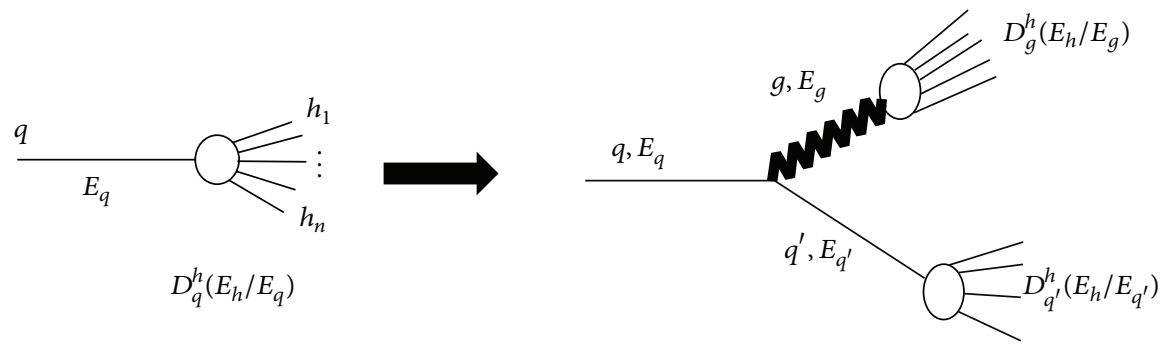

FIGURE 9: An overview of how a gluon radiation leads into scaling violation.

will be changed into the final hadrons and it is called the fragmentation function. As mentioned above, this feature shows the possibility of production hadron " $h$," with the energy scale of “ $x$," from quark " $q$." In general, " $D$ ” depends on the kind of initial quark, hadron, and the center of mass energy. Hadron production in the annihilation process of electron-positron can be expressed in the domain of structure functions of $F_{1}$ and $F_{2}$. Considering $F_{1}$ and $F_{2}$, differential cross section of Drell et al. [13], is presented as follows:

$$
\begin{aligned}
\frac{d^{2} \sigma}{d x d \cos \theta}= & \frac{3}{4} \sigma_{\circ} \cdot x \\
& \cdot \beta\left[2 F_{1}(x, s)+\frac{x \cdot \beta}{2} F_{2}(x, s) \cdot \sin ^{2} \theta\right],
\end{aligned}
$$

where $\sigma$ o shows the cross section of QED in the 0th order, $\theta$ is the angle of the proton momentum $P$ with respect to the jet axis, and $\beta=\left(1-E_{q} / E_{\text {jet }}\right)^{1 / 2} \cdot F_{1}$ and $F_{2}$ can be expressed in terms of the transverse and longitudinal structure function as follows:

$$
\begin{aligned}
& F_{T}(x, s)=2 F_{1}(x, s) \\
& F_{L}(x, s)=2 F_{1}(x, s)+F_{2}(x, s) ;
\end{aligned}
$$

then

$$
\begin{aligned}
\frac{d^{2} \sigma}{d x d \cos \theta}= & \frac{3}{4} \sigma_{\circ} \\
& \cdot x\left[F_{T} \cdot\left(1+\cos ^{2} \theta\right)+\frac{1}{2} F_{L} \cdot \sin ^{2} \theta\right] .
\end{aligned}
$$

In comparison with the observed scaling behavior in the space-shaped distribution which was accounted as one of the accepted evidences for partons' presence, Drell et al. assumed that $F_{1}$ and $F_{2}$ and also $F_{L}$ and $F_{T}$ are scaled as follows:

$$
s \longrightarrow \infty \longrightarrow F(x, s)=F(x) .
$$

In the framework of quark-parton model, photon is being coupled to a spin $1 / 2$ parton and

$$
\begin{aligned}
F_{L}(x) & =0 \\
x F_{T} & =3 \sum e_{q}^{2}\left[D_{q}(x)+D_{\bar{q}}(x)\right] .
\end{aligned}
$$

So, the scaling hypothesis shows that the fragmentation function " $D$ " must be independent from the center of mass energy. These formulas are not trusty in the framework of QCD. Gluon radiation leads into scaling violation and affects the longitudinal structure function. These changes are simple. Due to the radiation of a gluon, the energy of quark will decrease from $E_{q}$ to $E_{q^{\prime}}$. Hadronic features of quark are being presented by a functional scale, $x^{\prime}=E_{h} / E_{q^{\prime}}$, instead of $x=$ $E_{h} / E_{q}$ and, for this reason, this quark will gain a momentum as its value in comparison to the evaluation of quark and parton will decrease (Figure 9).

So, the scaling violation of QCD causes the increase of particles production in the lower $x$ interval. In addition, the effects of gluon change the features of angular momentum of the array of partons and it leads into a longitudinal element of $F_{L}$. Figures 10 and 11 show the number of charged particles for jets of quarks and gluons, respectively, in which their $x_{E}$ is between $a$ and $b$, where $a$ and $b$ are two desired numbers between 0 and 1 . In these two figures the results related to scaling violations of fragmentation functions of quark and gluon jets along with the other results and AMY data are presented. These results are gained by considering the events of three jets.

As can be seen from these figures, the probability for producing low energy charged particles is more than the probability for those that are produced with higher $x_{E}$. This means that production of particles which carry high amount of total energy of event is ignorable and more particles only carry minor energy with respect to energy of event. Also, there are three types of NLO related to low energy and mediate and high energy events. As our models are constructed in terms of properties of quantum mechanics and these properties are different in low and high energies, we have to use three models. As we can see, data in low energy are more in agreement with BFGW and $\mathrm{Kr}$ and in agreement with KKP in high energy. For the fragmentation functions of gluon jet (Figure 11), data explanation by NLO evaluations is not good for description. So, based on Figures 10 and 11, scaling violation is observable as these violations for small $x_{E}$ are along with the positive slope and for large $x_{E}$ are along with the negative slope. In other words, it is being expected that stronger scaling violations will occur in the gluon jets to the quark ones. It is clear that the data in the low range of $x_{E}$ are under KKP evaluations. So in this area, the data is consistent with the evaluations of $\mathrm{Kr}$ and BFGW models. For the large values of $x_{E}$, data is consistent with KKP evaluations. In Figures 12 and 13, the dependency of the fragmentation functions of quark and gluon jets to $x_{E}$ for AMY data was 


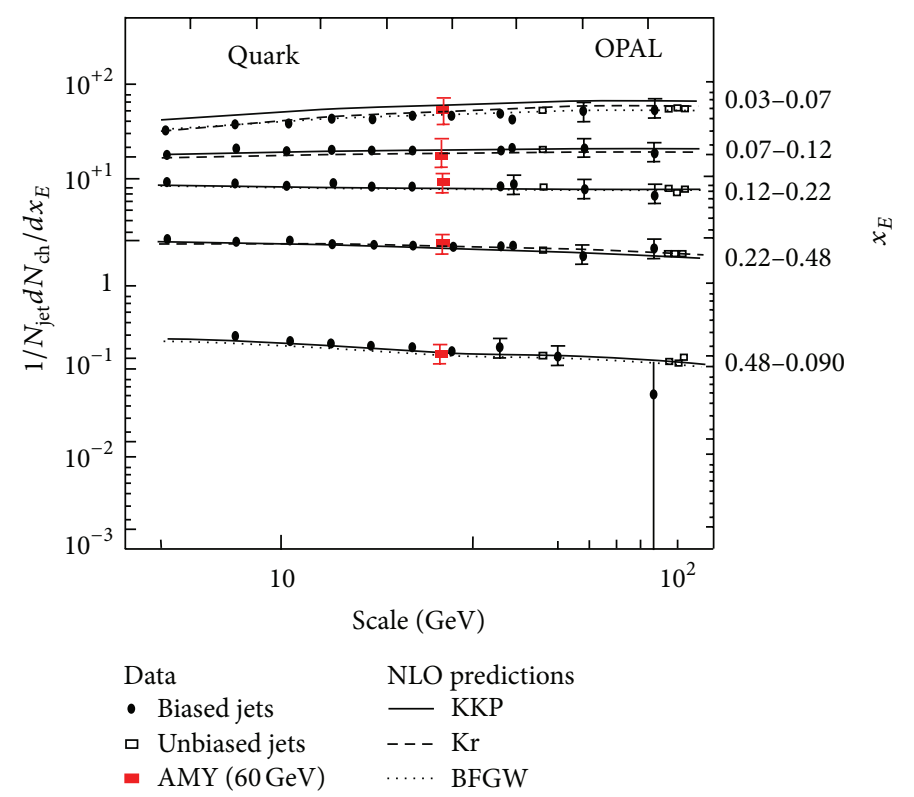

FIGURE 10: Scale dependence of the quark fragmentation function in different $x_{E}$ 's intervals [12-15].

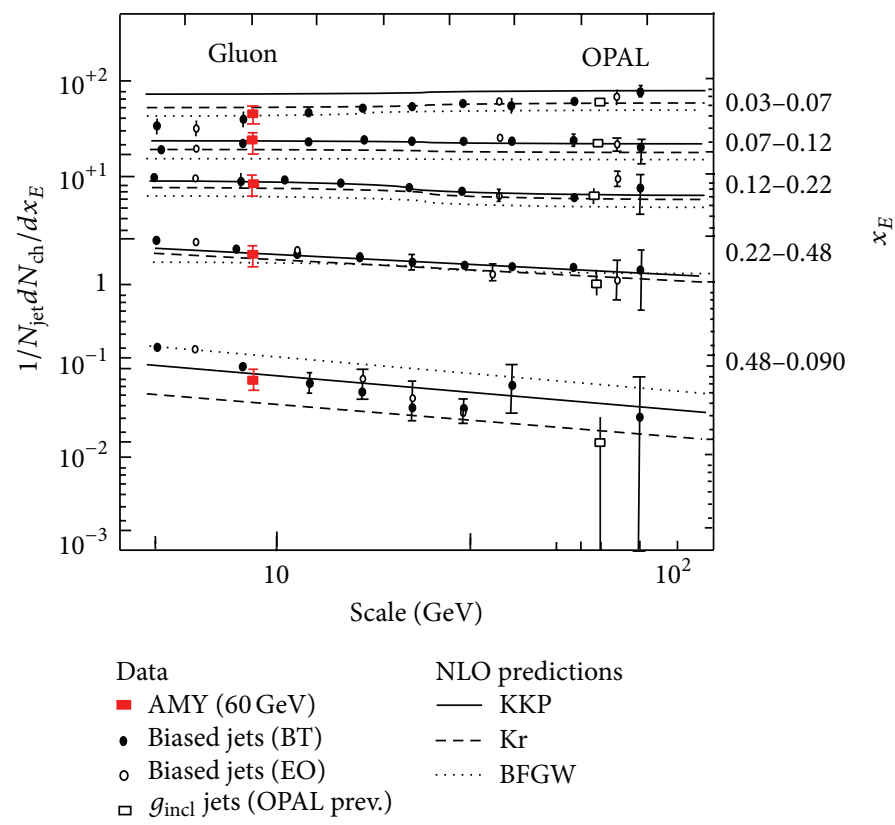

Figure 11: Scale dependence of the gluon fragmentation function in different $x_{E}$ 's intervals [12-15].

shown with the other results. In these two figures, AMY data in small $x_{E}$ is consistent with the theoretical evaluations and also with the other data from other energies. By increasing $x_{E}$, the cross section shows its descending trend as this trend is different from the fragmentation functions of gluon jets for various energies. So, by increasing energy, the dependency of cross section of these jets to energy will be increased. And for this reason, the scaling violation of fragmentation functions of gluon jets to the quark ones in the high energies is stronger.

\section{Conclusion}

In this paper, we have used data obtained from the annihilation process of electron-positron in AMY detector at $60 \mathrm{Gev}$ center of mass energy and, at first, the momentum spectrum of charged particles to the jet axis is studied as the sphericity axis was selected as the jet axis. Then, we notice that the cross section of particles production was decreased by increasing the momentum. In a wide range of energies, a considerable increase of average of squared momentum occurs with 

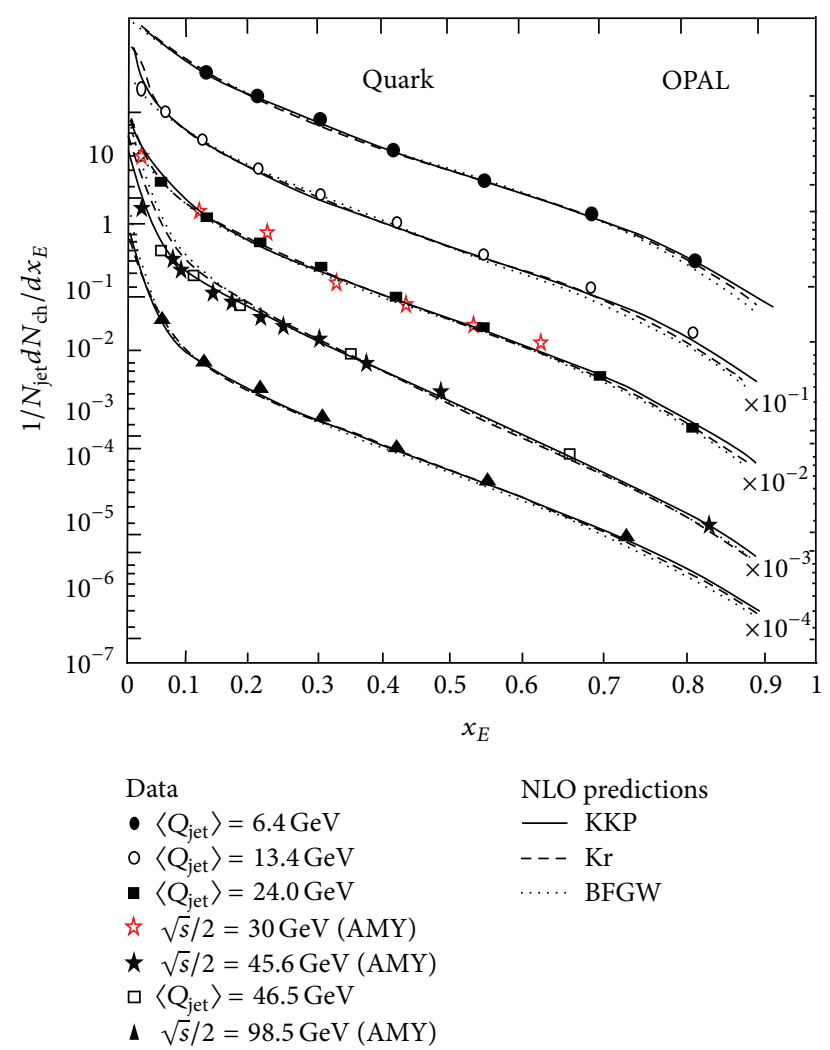

FIGURE 12: Dependency of the fragmentation functions of quark jets to $x_{E}$ in different scales at different c.m. energies [12-15].

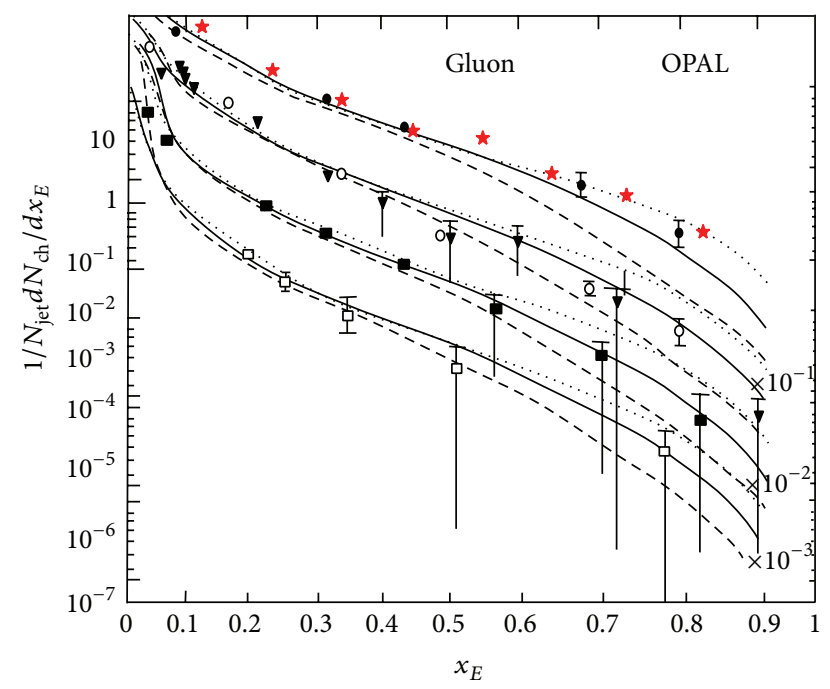

$$
\begin{array}{ll}
\text { Data } & \text { NLO predictions } \\
\star \sqrt{s} / 2=30 \mathrm{GeV}(\mathrm{AMY}) & -\mathrm{KKP} \\
\text { - }\left\langle\mathrm{Q}_{\text {jet }}\right\rangle=6.4 \mathrm{GeV} & --\mathrm{Kr} \\
\circ\left\langle\mathrm{Q}_{\text {jet }}\right\rangle=13.4 \mathrm{GeV} & \cdots \cdots \mathrm{BFGW} \\
\text { \ } E_{\text {jet }}=14.24 \mathrm{GeV} \text { (OPAL prev.) } & \\
\text { - }\left\langle\mathrm{Q}_{\text {jet }}\right\rangle=24.0 \mathrm{GeV} & \\
\text { - }\left\langle\mathrm{Q}_{\text {jet }}\right\rangle=48.5 \mathrm{GeV} &
\end{array}
$$

FIGURE 13: Dependency of the fragmentation functions of gluon jets to $x_{E}$ in different scales at different c.m. energies [12-15]. a linear trend by increasing the energy. Its reason is that the possibility of gluon radiation at high energies is high. In other words, scaling violation of fragmentation functions occurs at higher energies with higher probability. It is being observed that, in the large PT regime, by increasing the center of mass energy, " $W$," the number of particles is increased as the radiation of gluon could be a main reason for such case. In other words, radiation of gluon affects the flux of particles in this domain. And also the distribution of fragmentation functions is being studied. In addition, the violations of scaling are considerable in these distributions. These violations for the fragmentation functions of gluon jets to the quark ones, in the high energies, were much more considerable. Finally, the reason of the scaling violations in the fragmentation functions by increasing energy is determined as related to the fact that the possibility of gluon radiation at high energy is great. This result is consistent with the prediction of QCD.

\section{Competing Interests}

The author declares no competing interests regarding this paper.

\section{Acknowledgments}

The author would like to thank Islamic Azad University for fruitful collaborations. Financial support of this study has been made by Islamic Azad University, Shiraz branch.

\section{References}

[1] K. Geiger, "Space-time description of ultra-relativistic nuclear collisions in the QCD parton picture," Physics Reports, vol. 258, no. 4-5, pp. 237-376, 1995.

[2] K. Konishi, A. Ukawa, and G. Veneziano, "A simple algorithm for QCD jets," Physics Letters B, vol. 78, no. 2-3, pp. 243-248, 1978.

[3] DELPHI Collaboration, "Measurement of the gluon fragmentation function and a comparison of the scaling violation in gluon and quark jets," The European Physical Journal C, vol. 13, no. 4, pp. 573-589, 2000.

[4] J. Binnewies, B. A. Kniehl, and G. Kramer, "Next-to-leading order fragmentation functions for pions and kaons," Zeitschrift für Physik C, vol. 65, no. 3, pp. 471-480, 1995.

[5] X. Ji, J. Ma, and F. Yuan, "QCD factorization for semi-inclusive deep-inelastic scattering at low transverse momentum," Physical Review D, vol. 71, no. 3, Article ID 034005, 2005.

[6] J. Collins, Foundations of Perturbative QCD, Cambridge University Press, 2013.

[7] S. M. Aybat and T. C. Rogers, "Transverse momentum dependent parton distribution and fragmentation functions with QCD evolution," Physical Review D, vol. 83, no. 11, Article ID 114042, 2011.

[8] W. Thome, K. Eggert, K. Giboni et al., "Charged particle multiplicity distributions in pp collisions at ISR energies," Nuclear Physics B, vol. 129, no. 3, pp. 365-389, 1977.

[9] J. Binnewies, B. A. Kniehl, and G. Kramer, "Inclusive hadron production in photon-photon collisions at next-to-leading order," Physical Review D-Particles, Fields, Gravitation and Cosmology, vol. 53, no. 11, pp. 6110-6119, 1996. 
[10] A. Sepehri, T. Ghaffary, and M. E. Zomorrodian, "Measurement of strong coupling constant from transverse momentum," Canadian Journal of Physics, vol. 87, no. 11, pp. 1151-1158, 2009.

[11] K. Aamodt, N. Abel, U. Abeysekara et al., "Transverse momentum spectra of charged particles in proton-proton collisions at $\sqrt{s}=900 \mathrm{Gev}$ with ALICE at the LHC," Physics Letters B, vol. 693, no. 2, pp. 53-68, 2010.

[12] D. P. Anderle, F. Ringer, and M. Stratmann, "Fragmentation functions at next-to-next-to-leading order accuracy," Physical Review D, vol. 92, no. 11, Article ID 114017, 2015.

[13] S. D. Drell, D. J. Levy, and T. Yan, “Theory of deep-inelastic lepton-nucleon scattering and lepton-pair annihilation processes. I," Physical Review, vol. 187, no. 5, pp. 2159-2171, 1969.

[14] S. Kretzer, "Fragmentation functions from flavor-inclusive and flavor-tagged $\mathrm{e}^{+} \mathrm{e}^{-}$annihilations," Physical Review D, vol. 62, no. 5, Article ID 054001, 2000.

[15] L. Bourhis, M. Fontannaz, J. P. Guillet, and M. Werlen, "Nextto-leading order determination of fragmentation functions," European Physical Journal C, vol. 19, no. 1, pp. 89-98, 2001.

[16] G. Aad, E. Abat, B. Abbott et al., "Charged-particle multiplicities in $p p$ interactions at $\sqrt{s}=900 \mathrm{Gev}$ measured with the ATLAS detector at the LHC," Physics Letters B, vol. 688, no. 1, pp. 21-42, 2010.

[17] R. E. Ansorge, B. Åsman, L. Burow et al., "Charged particle multiplicity distributions at 200 and $900 \mathrm{GeV}$ c.m. energy," Zeitschrift für Physik C: Particles and Fields, vol. 43, no. 3, pp. 357-374, 1989.

[18] P. Slattery, "Evidence for the onset of semi-inclusive scaling in proton-proton collisions in the $50-300-\mathrm{GeV} / c$ momentum range," Physical Review Letters, vol. 29, no. 24, pp. 1624-1627, 1972.

[19] F. Rimondi and CDF Collaboration, "Multiplicity distributions in $\bar{p} p$ interactions at $\sqrt{s}=1800 \mathrm{GeV}$," in Proceedings of the 23th International Symposium on Multiparticle Dynamics, pp. 400404, Aspen, Colo, USA, September 1993.

[20] R. P. Feynman, "Very high-energy collisions of hadrons," Physical Review Letters, vol. 23, no. 24, pp. 1415-1417, 1969.

[21] Z. Koba, H. B. Nielsen, and P. Olesen, "Scaling of multiplicity distributions in high energy hadron collisions," Nuclear Physics $B$, vol. 40, pp. 317-334, 1972.

[22] A. H. Mueller, "On the multiplicity of hadrons in QCD jets," Physics Letters B, vol. 104, no. 2, pp. 161-164, 1981.

[23] A. H. Mueller, "Multiplicity and hadron distributions in QCD jets: non-leading terms," Nuclear Physics B, vol. 213, no. 1, pp. 85-108, 1983.

[24] A. Breakstone, R. Campanini, H. B. Crawley et al., "Charged multiplicity distribution in pp interactions at CERN ISR energies," Physical Review D, vol. 30, no. 3, pp. 528-535, 1984.

[25] S. Catani, Y. L. Dokshitzer, M. Olsson, G. Turnock, and B. R. Webber, "New clustering algorithm for multijet cross sections in $\mathrm{e}^{+} \mathrm{e}^{-}$annihilation," Physics Letters B, vol. 269, no. 3-4, pp. 432438, 1991.

[26] S. Bethke, J. Allison, K. Ambrus et al., "Experimental investigation of the energy dependence of the strong coupling strength," Physics Letters B, vol. 213, no. 2, pp. 235-241, 1988.

[27] J. F. Donoghue, F. E. Low, and S.-Y. Pi, "Tensor analysis of hadronic jets in quantum chromodynamics," Physical Review D, vol. 20, no. 11, pp. 2759-2762, 1979.

[28] M. Althoff, W. Braunschweig, F. J. Kirschfink et al., "Jet production and fragmentation ine ${ }^{+} \mathrm{e}^{-}$annihilation at $12-43 \mathrm{GeV}$," Zeitschrift für Physik C Particles and Fields, vol. 22, no. 4, pp. 307-340, 1984.
[29] R. Perez-Ramos and D. d'Enterria, "Energy evolution of the moments of the hadron distribution in QCD jets including NNLL resummation and NLO running-coupling corrections," Journal of High Energy Physics, vol. 2014, article 68, 2014. 

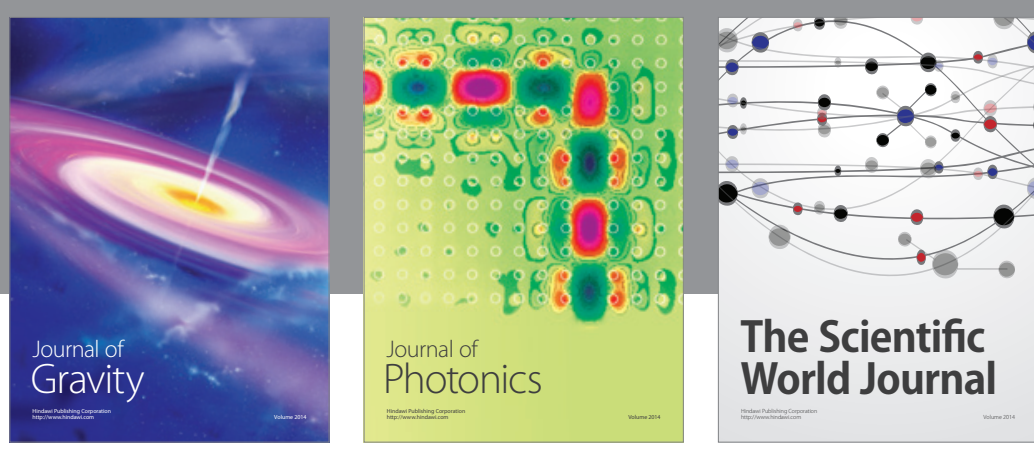

The Scientific World Journal
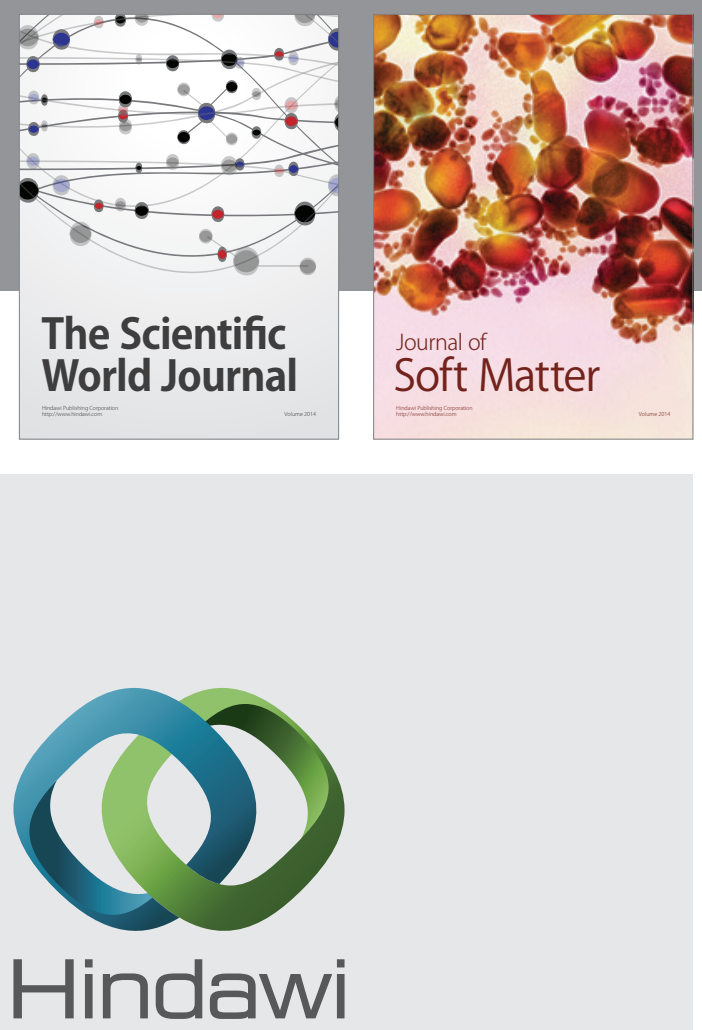

Submit your manuscripts at

http://www.hindawi.com

nternational Journal of

Statistical Mechanics
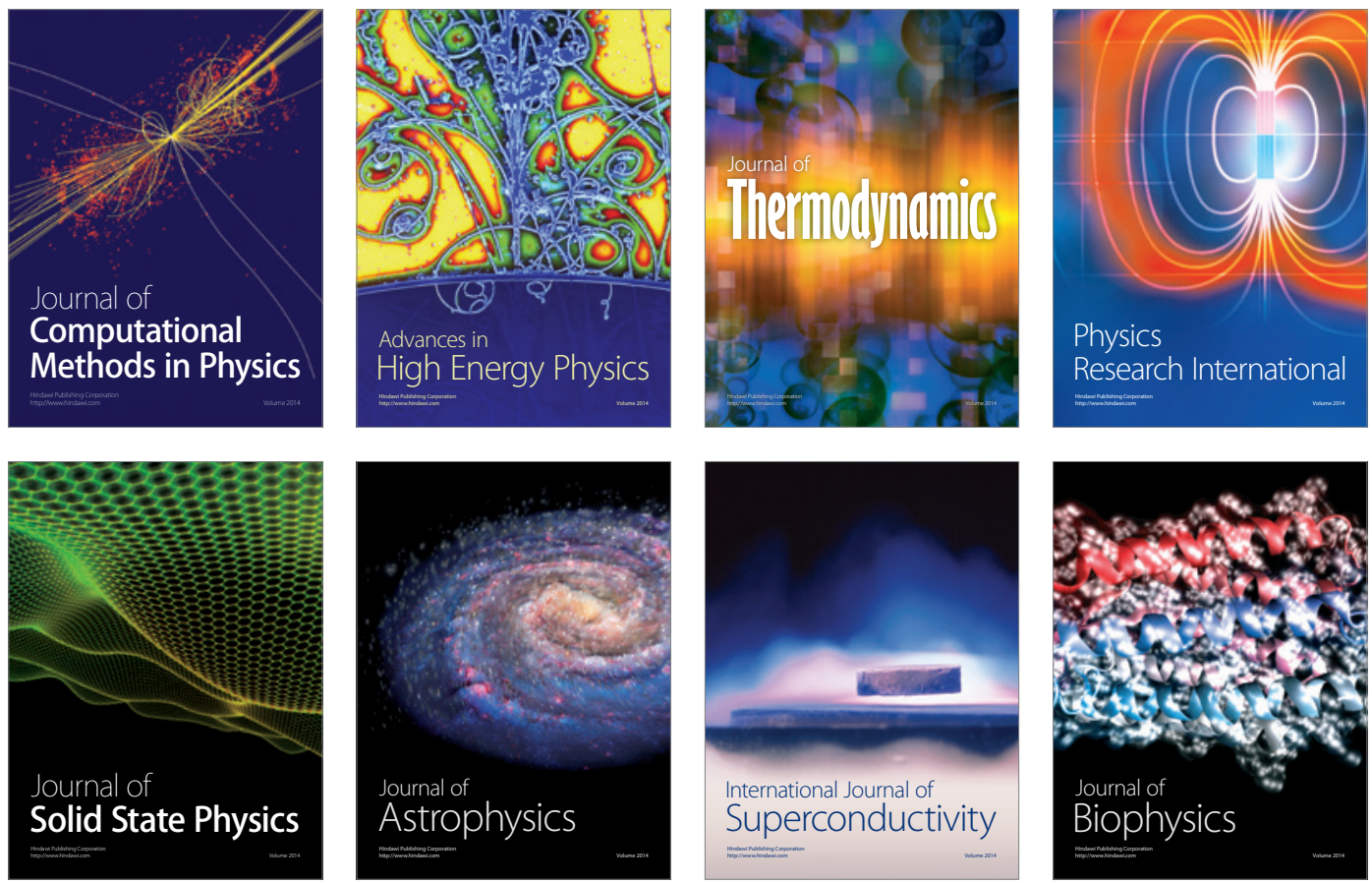
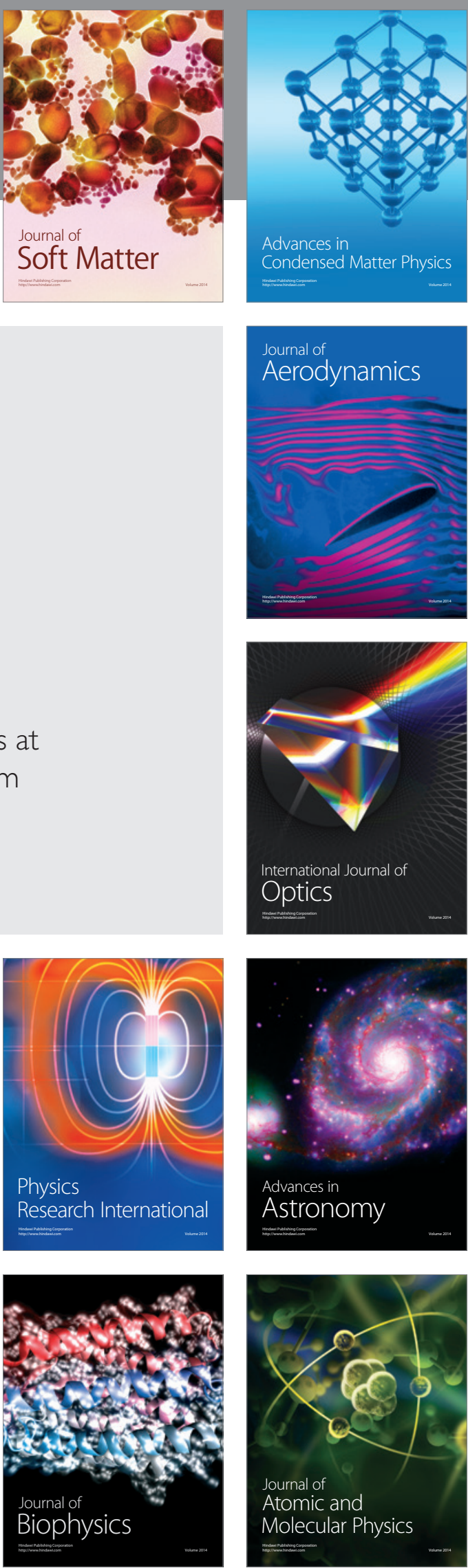